\title{
Compositions of Random Functions on a Finite Set
}

\author{
Avinash Dalal \\ MCS Department, Drexel University \\ Philadelphia, Pa. 19104 \\ ADalal@drexel.edu
}

\author{
Eric Schmutz \\ Drexel University and Swarthmore College \\ Philadelphia, Pa., 19104 \\ Eric.Jonathan.Schmutz@drexel.edu
}

Submitted: July 21, 2001; Accepted: July 9, 2002

MR Subject Classifications: 60C05, 60J10, 05A16, 05A05

\begin{abstract}
If we compose sufficiently many random functions on a finite set, then the composite function will be constant. We determine the number of compositions that are needed, on average. Choose random functions $f_{1}, f_{2}, f_{3}, \ldots$ independently and uniformly from among the $n^{n}$ functions from $[n]$ into $[n]$. For $t>1$, let $g_{t}=f_{t} \circ f_{t-1} \circ \cdots \circ f_{1}$ be the composition of the first $t$ functions. Let $T$ be the smallest $t$ for which $g_{t}$ is constant(i.e. $g_{t}(i)=g_{t}(j)$ for all $\left.i, j\right)$. We prove that $E(T) \sim 2 n$ as $n \rightarrow \infty$, where $E(T)$ denotes the expected value of $T$.
\end{abstract}

\section{Introduction}

If we compose sufficiently many random functions on a finite set then the composite function is constant. We ask how long this takes, on average. More precisely, let $U_{n}$ be the set of $n^{n}$ functions from $[n]$ to $[n]$. Let $A_{n}$ be the $n$ element subset of $U_{n}$ consisting of the constant functions: $g \in A_{n}$ iff $g(i)=g(j)$ for all $i, j$. Let $f_{1}, f_{2}, f_{3}, \ldots$ be a sequence of random functions chosen independently and uniformly from $U_{n}$. Let $g_{1}=f_{1}$, and for $t>1$ let $g_{t}=f_{t} \circ g_{t-1}$ be the composition of the first $t$ random maps. Define $T\left(\left\langle f_{i}\right\rangle_{i=1}^{\infty}\right)$ to be the smallest $t$ for which $g_{t} \in A_{n}$. (If no such $t$ exists, define $T=\infty$. It is not difficult to show that $\operatorname{Pr}(T=\infty)=0$.) Our goal in this paper is to estimate $E(T)$.

It is natural to restate the problem as a question about a Markov chain. The state space is $\mathcal{S}=\left\{s_{1}, s_{2}, \ldots, s_{n}\right\}$. For $t>0$ and $r \in[n]$, we are in state $s_{r}$ if and only if $g_{t}$ has exactly $r$ elements in its range. With the convention that $g_{0}$ is the identity permutation, we start in state $s_{n}$ at time $t=0$. The question is how long (i.e. how many compositions) it takes to reach the absorbing state $s_{1}$.

For $m>1$, let $\tau_{m}=\left|\left\{t:\left|\operatorname{Range}\left(g_{t}\right)\right|=m\right\}\right|$ be the amount of time we are in state $s_{m}$. Thus $T=\sum_{m=2}^{n} \tau_{m}$. Let $\mathcal{T}$ consist of those states that are actually visited: 
for $m>1, s_{m} \in \mathcal{T}$ iff $\tau_{m}>0$. The visited states $\mathcal{T}$ are a (non-uniform) random subset of $\mathcal{S}$ that includes at least two elements, namely $s_{n}$ and (with probability 1 ) $s_{1}$. We prove later that $\mathcal{T}$ typically contains most of the small numbered states and relatively few of the large numbered states. This observation forms the basis for our proof of

Theorem $1 E(T)=2 n(1+o(1))$ as $n \rightarrow \infty$.

We should mention that there is a standard approach to our problem using the transition matrix $P$ and linear algebra. Let $Q$ be the matrix that is obtained from $P$ by striking out the first row and column of $P$. Then $E(T)$ is exactly the sum of the entries in the last row of $(I-Q)^{-1}$. See, for example, chapter 3 of [5]. This fact is very convenient if one wishes to compute $E(T)$ for specific small values of $n$. An anonymous referee conjectured that $E(T)=2 n-3+o(1)$ after observing that, for small values of $n,|E(T)-2 n+3| \leq 1$. This conjecture is plausible, but we are nowhere near a proof.

\section{The Transition Matrix}

The $n \times n$ transition matrix $P$ can be determined quite explicitly. Suppose $g_{t-1}$ has $i$ elements in its range, How many functions $f$ have the property that $f \circ g_{t-1}$ has exactly $j$ elements in its range? There are $\left(\begin{array}{c}n \\ j\end{array}\right)$ ways to choose the $j$-element range of $f \circ g_{t-1}$, and $S(i, j) j$ ! ways to map the $i$-element range of $g_{t-1}$ onto a given $j$ element set. (Here $S(i, j)$ is the number of ways to partition an $i$ element set into $j$ disjoint subsets, a Stirling number of the second kind.) Finally, there are $n-i$ elements in the complement of the range of $g_{t-1}$, and $n^{n-i}$ ways to map them into $[n]$. Thus there are $\left(\begin{array}{c}n \\ j\end{array}\right) S(i, j) j ! n^{n-i}$ functions $f$ with the desired property, and for $1 \leq i, j \leq n$, the transition matrix for the chain has $i, j$ 'th entry

$$
P(i, j)=\left(\begin{array}{l}
n \\
j
\end{array}\right) \frac{S(i, j) j !}{n^{i}} .
$$

The stationary distribution $\pi$ assigns probability 1 to $s_{1}$. The transition matrix has some nice properties. It is lower triangular, which means the eigenvalues are just the diagonal entries: for $1 \leq m \leq n$,

$$
\lambda_{m}=P(m, m)=\prod_{k=0}^{m-1}\left(1-\frac{k}{n}\right) .
$$

For future reference we record two simple estimates for the eigenvalues, both of which follow easily from (2).

\section{Lemma 2}

$$
\lambda_{m}=1-\frac{\left(\begin{array}{c}
m \\
2
\end{array}\right)}{n}+O\left(\frac{m^{4}}{n^{2}}\right)
$$

and

$$
\lambda_{m} \leq \exp \left(-\left(\begin{array}{c}
m \\
2
\end{array}\right) / n\right) \text {. }
$$




\section{Lower Bound}

The proof of the lower bound requires an estimate for the Stirling numbers $S(m, k)$. The literature contains many precise but complicated estimates for these numbers. Here we prove a crude inequality whose simplicity makes it convenient for our purposes.

Lemma 3 For all positive integers $m$ and $k, S(m, k) \leq(2 k)^{m}$.

Proof: The proof of this lemma will be done by induction using the recurrence $S(m, k)=S(m-1, k-1)+k S(m-1, k)$. When $k=1$, we know that $S(m, 1)=1$ and $(2 k)^{m}=2^{m}$. So clearly the inequality holds true for $k=1$ (for all positive integers $m)$.

Now let $\phi_{m}$ denote the following statement: for all $k>1, S(m, k) \leq(2 k)^{m}$. It suffices to prove that $\phi_{m}$ is true for all $m$. For $m=1, S(1, k)=0 \leq 2 k$ for all $k>1$. Now let $k>1$ and assume, inductively, that $\phi_{m-1}$ is true (i.e. $S(m-1, k) \leq(2 k)^{m-1}$ for $k>1$.) Then we have

$$
\begin{gathered}
S(m, k)=S(m-1, k-1)+k S(m-1, k) \leq(2(k-1))^{m-1}+k(2 k)^{m-1} \\
=(2 k)^{m}\left\{\frac{1}{2}+\frac{(k-1)^{m-1}}{2 k^{m}}\right\} .
\end{gathered}
$$

Realize that the quantity inside the large braces is less than one.

With lemma 3 available, we can proceed with the proof that $E(T) \geq 2 n(1+o(1))$. Since $T=\sum_{m=2}^{n} \tau_{m}$, we have

$$
E(T)=\sum_{m=2}^{n} \operatorname{Pr}\left(s_{m} \in \mathcal{T}\right) E\left(\tau_{m} \mid s_{m} \in \mathcal{T}\right) .
$$

Obviously a lower bound is obtained by truncating this sum. To simplify notation, let $\ell=\lfloor\log \log n\rfloor$. Then

$$
E(T) \geq \sum_{m=2}^{\ell} \operatorname{Pr}\left(s_{m} \in \mathcal{T}\right) E\left(\tau_{m} \mid s_{m} \in \mathcal{T}\right) .
$$

To estimate the second factor in each term of (4), note that

$$
E\left(\tau_{m} \mid s_{m} \in \mathcal{T}\right)=\sum_{t=1}^{\infty} t \lambda_{m}^{t-1}\left(1-\lambda_{m}\right)=\frac{1}{1-\lambda_{m}} .
$$

Applying lemma 2, we get

$$
E\left(\tau_{m} \mid s_{m} \in \mathcal{T}\right)=\frac{n}{\left(\begin{array}{c}
m \\
2
\end{array}\right)}\left(1+O\left(\frac{m^{2}}{n}\right)\right) .
$$

To estimate the first factor of each term in (4), we make the following observation: if $s_{m} \notin \mathcal{T}$, then there is a transition from $s_{m+d}$ to $s_{m-j}$ for some positive integers $d$ and $j$. Hence, 


$$
\operatorname{Pr}\left(s_{m} \notin \mathcal{T}\right)=\sum_{d=1}^{n-m} \sum_{j=1}^{m-1} \operatorname{Pr}\left(s_{m+d} \in \mathcal{T}\right) \frac{P(m+d, m-j)}{\left(1-\lambda_{m+d}\right)} .
$$

(The factor $\left(1-\lambda_{m+d}\right)^{-1}=\sum_{i=0}^{\infty} P(m+d, m+d)^{i}$ is there because we remain in state $s_{m+d}$ for some number of transitions $i \geq 0$ before moving on to state $s_{m-j}$.)

Let $\sigma:=\sum_{d=1}^{n-m} \sum_{j=1}^{m-1} \frac{S(m+d, m-j)}{n^{j+d}} \frac{\lambda_{m-j}}{1-\lambda_{m+d}}$. Putting (1) and $\operatorname{Pr}\left(s_{m+d} \in \mathcal{T}\right) \leq 1$ into (7), we get

$$
\operatorname{Pr}\left(s_{m} \notin \mathcal{T}\right) \leq \sum_{d=1}^{n-m} \sum_{j=1}^{m-1} 1 \cdot\left(\begin{array}{c}
n \\
m-j
\end{array}\right) \frac{S(m+d, m-j)(m-j) !}{n^{m+d}\left(1-\lambda_{m+d}\right)}=\sigma .
$$

A first step in bounding $\sigma$ is to note that $1>\left(1-\frac{1}{n}\right)=\lambda_{2} \geq \lambda_{3} \geq \lambda_{4} \geq \ldots \geq \lambda_{n}>0$, and therefore

$$
\frac{\lambda_{m-j}}{1-\lambda_{m+d}} \leq \frac{1}{1-\lambda_{m+d}} \leq \frac{1}{1-\lambda_{2}}=n-1
$$

Hence

$$
\sigma \leq(n-1) \sum_{d=1}^{n-m} \frac{1}{n^{d}} \sum_{j=1}^{m-1} \frac{S(m+d, m-j)}{n^{j}} .
$$

Applying lemma 3 to each term of the inside sum, we get

$$
\begin{gathered}
\sum_{j=1}^{m-1} \frac{S(m+d, m-j)}{n^{j}} \leq \sum_{j=1}^{m-1} \frac{(2(m-j))^{m+d}}{n^{j}} \\
\leq \frac{m(2 m-2)^{m+d}}{n}<\frac{\ell(2 \ell)^{\ell+d}}{n}
\end{gathered}
$$

Hence

$$
\sigma \leq(n-1) \frac{\ell(2 \ell)^{\ell}}{n} \sum_{d=1}^{n-m}\left(\frac{2 \ell}{n}\right)^{d}=O\left(\frac{(2 \ell)^{\ell+2}}{n}\right)=o(1) .
$$

Thus $\operatorname{Pr}\left(s_{m} \in \mathcal{T}\right) \geq 1-o(1)$ for all $m \leq \ell$, Putting this and (6) back into (4), and using the fact that $\sum_{m=2}^{\ell} \frac{1}{\left(\begin{array}{c}m \\ 2\end{array}\right)}=\sum_{m=2}^{\ell}\left(\frac{2}{m-1}-\frac{2}{m}\right)=2-\frac{2}{\ell}$, we get the lower bound $E(T) \geq 2 n(1+o(1))$.

\section{Upper Bound}

If $\left|\operatorname{Range}\left(g_{t-1}\right)\right|=m$, then the restriction of $f_{t}$ to $\operatorname{Range}\left(g_{t-1}\right)$ is a random function from an $m$ element set to $[n]$. Before proving that $E(T) \leq 2 n(1+o(1))$, we gather a simple lemma about the size of the size of the range for such random maps.

Lemma 4 Suppose $h:[m] \rightarrow[n]$ is selected uniformly at random from among the $n^{m}$ functions from $[m]$ into $[n]$, and let $R$ be the cardinality of the range of $h$. Then the mean and variance of $R$ are respectively $E(R)=n-n\left(1-\frac{1}{n}\right)^{m}$ and $\operatorname{Var}(R)=n^{2}\left\{\left(1-\frac{2}{n}\right)^{m}-\left(1-\frac{1}{n}\right)^{2 m}\right\}+n\left\{\left(1-\frac{1}{n}\right)^{m}-\left(1-\frac{2}{n}\right)^{m}\right\}$. 
Proof: Let $U=n-R=\sum_{i=1}^{n} I_{i}$, where $I_{i}$ is 1 if $i$ is not in the range of $h$, and otherwise $I_{i}$ is zero. Then $E(R)=n-E(U)$, and $\operatorname{Var}(R)=\operatorname{Var}(U)$.

$$
\begin{aligned}
& E(U)=n E\left(I_{1}\right)=n\left(1-\frac{1}{n}\right)^{m} . \\
& E\left(U^{2}\right)=\sum_{i \neq j} E\left(I_{i} I_{j}\right)+E(U) \\
& =n(n-1)\left(1-\frac{2}{n}\right)^{m}+E(U) .
\end{aligned}
$$

Therefore

$$
\operatorname{Var}(U)=n^{2}\left\{\left(1-\frac{2}{n}\right)^{m}-\left(1-\frac{1}{n}\right)^{2 m}\right\}+n\left\{\left(1-\frac{1}{n}\right)^{m}-\left(1-\frac{2}{n}\right)^{m}\right\} .
$$

The next corollary shows that there are gaps between the large states in $\mathcal{T}$. Let $\xi_{2}=\left\lfloor\frac{n}{\log ^{2} n}\right\rfloor$, and let $\beta=\beta(n)=\frac{1}{2}\left(\xi_{2}-n+n\left(1-\frac{1}{n}\right)^{\xi_{2}}\right)$. Although $\beta$ is quite large $\left(\beta \gg \frac{n}{\log ^{4} n}\right)$ all we really need for our purposes is that $\beta \rightarrow \infty$ as $n \rightarrow \infty$.

Corollary $5 \operatorname{Pr}\left(s_{m-\delta} \notin \mathcal{T}\right.$ for $\left.1 \leq \delta \leq \beta \mid s_{m} \in \mathcal{T}\right)=1-o(1)$ uniformly for $\xi_{2} \leq m \leq n$.

Proof: Suppose we are in state $s_{m}$ at time $t-1$ and select the next function $f_{t}$. Let $h$ be the restriction of $f_{t}$ to the range of $g_{t-1}$, and let $R$ be the cardinality of the range of $h$, and let $B=m-R$. Observe that if $B>\beta$ then the next $\beta$ states are missed: $s_{m-\delta} \notin \mathcal{T}$ for $1 \leq \delta \leq \beta$. Note that $E(B)=m-n+n\left(1-\frac{1}{n}\right)^{m}>2 \beta$. Applying Chebyshev's inequality to the random variable $B$, we get

$$
\operatorname{Pr}(B \leq \beta) \leq \operatorname{Pr}\left(B \leq \frac{1}{2} E(B)\right) \leq \frac{4 \operatorname{Var}(B)}{(E(B))^{2}} .
$$

For $\xi_{2} \leq m \leq n$, we have $E(B)=m-n+n\left(1-\frac{1}{n}\right)^{m} \geq \xi_{2}-n+n\left(1-\frac{1}{n}\right)^{\xi_{2}} \gg \frac{n}{\log ^{4} n}$. (A calculus exercise shows that $E(B)$ is an increasing function of $m$.) To bound $\operatorname{Var}(B)$ note that,

$$
\left(1-\frac{2}{n}\right)^{m}-\left(1-\frac{1}{n}\right)^{2 m}=O\left(\frac{m}{n^{2}}\right)
$$

Therefore (10) yields

$$
\operatorname{Pr}(B \leq \beta)=O\left(\frac{m \log ^{8} n}{n^{2}}\right)=o(1)
$$

Now we proceed with the proof of the upper bound $E(T) \leq 2 n(1+o(1))$. Split the sum (3) into three separate sums as follows. Let $\xi_{1}=\left\lfloor\sqrt{\frac{n}{\log n}}\right\rfloor$, and let $\xi_{2}=\left\lfloor\frac{n}{\log ^{2} n}\right\rfloor$, so that (3) becomes 


$$
E(T)=\sum_{m=2}^{\xi_{1}}+\sum_{m=\xi_{1}+1}^{\xi_{2}}+\sum_{m=\xi_{2}+1}^{n}
$$

The first sum in (11) is estimated using (5), lemma 2, and the fact that $\operatorname{Pr}\left(s_{m} \in\right.$ $\mathcal{T}) \leq 1$ :

$$
\begin{gathered}
\sum_{m=2}^{\xi_{1}} \operatorname{Pr}\left(s_{m} \in \mathcal{T}\right) E\left(\tau_{m} \mid s_{m} \in \mathcal{T}\right) \leq \sum_{m=2}^{\xi_{1}} \frac{1}{1-\lambda_{m}} \\
=\sum_{m=2}^{\xi_{1}} \frac{1}{\frac{\left(\begin{array}{c}
m \\
2
\end{array}\right)}{n}+O\left(\frac{m^{4}}{n^{2}}\right)} \\
=\left(1+O\left(\frac{\xi_{1}^{2}}{n}\right)\right) n \sum_{m=2}^{\xi_{1}} \frac{1}{\left(\begin{array}{c}
m \\
2
\end{array}\right)}=2 n(1+o(1)) .
\end{gathered}
$$

The second sum in (11) is estimated using a crude bound on the eigenvalues. For $\xi_{1}<m \leq \xi_{2}$, we have $\lambda_{m} \leq \lambda_{\xi_{1}}=1-\frac{1}{2 \log n}+O\left(\frac{1}{\sqrt{n \log n}}\right)$. Hence the second sum in (11) is at most

$$
\begin{gathered}
\sum_{m=\xi_{1}+1}^{\xi_{2}} \frac{1}{1-\lambda_{m}} \leq \frac{1}{1-\lambda_{\xi_{1}}} \sum_{m=\xi_{1}}^{\xi_{2}} 1 \\
=O\left(\xi_{2} \log n\right)=O\left(\frac{n}{\log n}\right) .
\end{gathered}
$$

For the last sum in (11), we can no longer get away with the trivial estimate $\operatorname{Pr}\left(s_{m} \in \mathcal{T}\right) \leq 1$. However now the size of the eigenvalues can be handled less carefully:

$$
\sum_{m=\xi_{2}+1}^{n} \operatorname{Pr}\left(s_{m} \in \mathcal{T}\right) \frac{1}{1-\lambda_{m}} \leq\left(\max _{m \geq \xi_{2}} \frac{1}{1-\lambda_{m}}\right)\left(\sum_{m=\xi_{2}}^{n} \operatorname{Pr}\left(s_{m} \in \mathcal{T}\right)\right) .
$$

The first factor in (12) is easily estimated using (2):

$$
\max _{m \geq \xi_{2}} \frac{1}{1-\lambda_{m}}=\frac{1}{1-\lambda_{\xi_{2}}} \leq \frac{1}{1-\exp \left(-\left(\begin{array}{c}
\xi_{2} \\
2
\end{array}\right) / n\right)} \leq 2
$$

for all sufficiently large $n$.

To deal with the second factor in (12) we use Corollary 5. The idea is that there cannot be too many "hits" (visited states) simply because every time there is a hit it is followed by $\beta$ "misses". To make this precise, define $V=\sum_{m=\xi_{2}}^{n} \chi_{m}$, where $\chi_{m}$ is 1 if $s_{m} \in \mathcal{T}$ and 0 otherwise. Thus the second factor in (12) is just $E(V)$. Also count large numbered states that are not in $\mathcal{T}$ with $W=\sum_{m=\xi_{2}}^{n}\left(1-\chi_{m}\right)$ so that $W+V=n+1-\xi_{2}$ and $E(V)=n+1-\xi_{2}-E(W)$. If a state $s_{m}$ is in $\mathcal{T}$, and if the next $\beta$ possible states $s_{m-1}, s_{m-2}, \ldots, s_{m-\beta}$ are not in $\mathcal{T}$, then those $\beta$ missed states together contribute exactly $\beta$ to $W$. 
If we let $J_{m}=\chi_{m} \cdot \prod_{\delta=1}^{\beta}\left(1-\chi_{m-\delta}\right)$, then $W \geq \beta \sum_{m \geq \xi_{2}} J_{m}$. But then

$$
\begin{gathered}
E(W) \geq \beta \sum_{m \geq \xi_{2}} E\left(J_{m}\right)= \\
\beta \sum_{m \geq \xi_{2}} \operatorname{Pr}\left(s_{m} \in \mathcal{T}\right) \operatorname{Pr}\left(s_{m-1}, s_{m-2}, \ldots s_{m-\beta} \notin \mathcal{T} \mid s_{m} \in \mathcal{T}\right) .
\end{gathered}
$$

By Corollary 5,

$$
\operatorname{Pr}\left(s_{m-1}, s_{m-2}, \ldots s_{m-\beta} \notin \mathcal{T} \mid s_{m} \in \mathcal{T}\right)=1-o(1) .
$$

Hence

$$
E(W) \geq \beta(1+o(1)) \sum_{m=\xi_{2}}^{n} \operatorname{Pr}\left(s_{m} \in \mathcal{T}\right)=(1+o(1)) \beta E(V) .
$$

But then

$$
\left.E(V)=n+1-\xi_{2}-E(W) \leq n+1-\xi_{2}-\beta(1+o(1))\right) E(V),
$$

which implies that

$$
E(V) \leq \frac{n+1-\xi_{2}}{1+\beta(1+o(1))}=O\left(\log ^{4} n\right)
$$

Thus the second factor of (12) is $o(n)$, which means that the third sum in (11) is negligible.

\section{References}

[1] D.Aldous and J.Fill, Reversible Markov Chains and Random Walks on Graphs" http://stat.berkeley.edu/users/aldous.

[2] P.Diaconis and D.Freedman, Iterated Random Functions, SIAM Review $4 \mathbf{1}$ No. $1, \mathrm{p} 45-76$.

[3] J.C.Hansen and J.Jaworski, Large Components of Random Mappings, Random Structures and Algorithms 17 (2000) 317-342.

[4] J.Kemeny, J.L.Snell, and A.W.Knapp, Denumerable Markov Chains, Van Nostrand Co., 1966.

[5] J.G.Kemeny, J.L.Snell, Finite Markov Chains, Springer Verlag, 1976.

[6] J.Jaworski, A Random Bipartite Mapping, Annals of Discrete Math., 28 137158 (1985).

[7] V.F.Kolchin, Random Mappings, Optimization Software, 1986.

[8] V.F.Kolchin, B.A.Sevastyanov, and V.P.Chistaykov, Random Allocations, Winston, 1978.

[9] J. S. Rosenthal, Convergence Rates for Markov Chains, SIAM Review 37 387405. 